\title{
The intersection of bronchoscopy and extracorporeal membrane oxygenation
}

\author{
Julie Lin ${ }^{1}$, Laura Frye ${ }^{2}$ \\ ${ }^{1}$ Department of Pulmonary of Critical Care Medicine, University of Chicago Medical Center, Chicago, IL, USA; ${ }^{2}$ Division of Allergy, Pulmonary, \\ and Critical Care Medicine, University of Wisconsin, Madison, WI, USA \\ Contributions: (I) Conception and design: None; (II) Administrative support: None; (III) Provision of study materials or patients: None; (IV) \\ Collection and assembly of data: None; (V) Data analysis and interpretation: None; (VI) Manuscript writing: Both authors; (VII) Final approval of \\ manuscript: Both authors. \\ Correspondence to: Laura Frye, MD. Division of Allergy, Pulmonary, and Critical Care Medicine, University of Wisconsin, 600 Highland Ave, \\ Madison, WI 53792, USA. Email: 1frye@medicine.wisc.edu.
}

\begin{abstract}
Central airway obstruction (CAO), which results from malignant, benign or iatrogenic etiologies, causes significant morbidity and mortality and can be seen in both the pediatric and adult patient population. Patients frequently present to the hospital with dyspnea, stridor, and respiratory distress, indicating impending respiratory failure. Heliox is used to help alleviate symptoms while procedural planning takes place. A multidisciplinary approach to airway management is often needed. Interventional pulmonologists treat $\mathrm{CAO}$ with rigid of flexible bronchoscopy in order to deliver therapeutic interventions under general anesthesia. In severe CAO where there is concern for total loss of the airway creating a life-threatening situation for the patient during procedural intervention, short term extracorporeal membrane oxygenation or ECMO has been successfully reported in the literature to provide ventilation and oxygenation support throughout the procedure. Venoarterial ECMO can be used to augment cardiac output in cases of central tumors with cardiac involvement. ECMO can also be used for the removal of tracheal stents when there is a concern that ventilation will be interrupted for a prolonged period of time. ECMO has also been reported as a salvage measure for patients with life threatening hemoptysis until more definitive interventions can be performed. Short term ECMO cannulation can be used with limited associated morbidity and a heparinfree approach can be pursued when there is a concern for bleeding. We will briefly review the anesthetic considerations in CAO as well as review cases of CAO where ECMO was employed to safely alleviate the airway compromise.
\end{abstract}

Keywords: Central airway obstruction (CAO); hemoptysis; extracorporeal membrane oxygenation (ECMO)

Submitted Jan 25, 2020. Accepted for publication Apr 29, 2020.

doi: 10.21037/jtd-2019-ipicu-08

View this article at: http://dx.doi.org/10.21037/jtd-2019-ipicu-08

Central airway obstruction (CAO) is caused by many disease processes including malignant, benign and iatrogenic etiologies leading to significant morbidity and mortality. The most common malignant cause of CAO is direct extension from tumor. Bronchogenic, esophageal and thyroid cancers are amongst the most frequent malignant CAOs. Primary tracheal tumors are relatively less common and are usually due to squamous cell cancer and adenoid cystic carcinoma. Common benign causes of CAO are due to stenosis from prior endotracheal or tracheostomy tubes and foreign bodies. Treatment of CAO is oftentimes performed by interventional pulmonologists (IPs) and management requires a multidisciplinary approach between IP, otolaryngologists, cardiothoracic surgeons, oncologists, and anesthesiologists (1).

Clinical signs and symptoms of CAO depend on the degree of airway narrowing. Patients will complain of dyspnea and wheezing with worsening nocturnal symptoms 
and when lying flat. Patients that develop stridor and are in tripod position have signs of severe stenosis and impending airway failure. They can present with respiratory distress or difficulty with ventilation. Symptoms typically do not manifest until the tracheal narrowing is less than $8 \mathrm{~mm}$ and can acutely worsen in the setting of infection due to mucosal inflammation. Flow volume loops will reveal fixed blunting of both the inspiratory and expiratory curves (1).

For a patient presenting with respiratory distress from $\mathrm{CAO}$, heliox can be used to alleviate symptoms and bridge to a more stable airway if needed. Its use for upper airway obstruction was first described in 1934 and has since been described in managing $\mathrm{CAO}$, asthma and chronic obstructive pulmonary disease (COPD) exacerbations. Heliox is a mixture of helium and oxygen, with helium at levels between $60-80 \%$ composition and is less dense than room air. Helium helps reduce turbulent flow and improve laminar flow and decrease resistance through a narrowed airway due to its relatively lower density than oxygen or nitrogen. Thus, flow is improved through a narrowed airway which can reduce work of breathing (1). In order for heliox to be effective, the oxygen concentration needs to be at the lowest possible level without sacrificing oxygenation (2). Heliox is delivered through a standard non-rebreather facemask or through noninvasive positive pressure mask. Heliox also aides in diffusing carbon dioxide 4-5 times faster than that of room air which is helpful in patients with acute hypercapnic respiratory failure. Heliox has very few complications since it is an inert gas-reported complications include hypothermia and hypoxemia. Hypothermia is due to the thermal conductivity of helium and warming the gas prevents this side effect. Hypoxemia is due to the need for lower concentrations of oxygen in order to have effective helium delivery (3).

While heliox is used to help relieve symptomatic CAO, it also provides a bridge to a therapeutic intervention that is necessary to relieve the obstruction. Prior to intervention, imaging modalities such as computed tomographic scans help characterize the degree and location of the obstruction and aide in procedural planning. Bronchoscopy is used to visualize the degree of obstruction. However, caution must be exercised when performing flexible bronchoscopy under moderate sedation. The relaxation of respiratory muscles can lead to loss of a patent but already narrowed airway and create a dangerous and life-threatening situation for the patient. Rigid bronchoscopy is the preferred procedure to secure a patient's airway and the CAO can be treated through the established passageway. Treatments for CAO such as dilations, coring, laser, and electrocautery can all be performed through the rigid bronchoscope and are discussed elsewhere (1).

Consultation with an anesthesiologist prior to performing therapeutic bronchoscopic interventions is necessary in order to devise a strategy to safely secure an airway. Patients should undergo a preoperative evaluation by anesthesia. Assessment includes a full history and examination, review of imaging studies, and lung function tests. Images are needed to characterize the severity of CAO. Any prior procedures involving anesthesia or endotracheal tube (ETT) size should be noted. Knowledge of oxygenation and ventilation status prior to the procedure is important given the higher risk nature during anesthesia administration.

There are multiple different anesthetic strategies for CAO reported in the literature; however, there is a common theme among them which is highlighted here. Premedication such as benzodiazepines should generally be avoided due to risk of airway compromise and hypoventilation. Anticholinergic agents such as atropine or glycopyrrolate should be given to dry up secretions that could obstruct the airway. There are various proposed ways in the literature for induction to secure airway. Topical anesthesia, inhalational anesthetic, intravenous (IV) agents and muscle relaxants are all proposed methods. The goal is to not induce coughing or laryngeal spasms when securing the airway because these episodes can worsen obstruction and create a life-threatening scenario. Caution should be used when applying topical or inhalational anesthetic as these agents can induce coughing episodes. Oral airways should not be inserted during light sedation since these can induce coughing as well. For procedures performed with flexible bronchoscopy, fiberoptic intubation is preferred to directly visualize and avoid trauma to the obstruction once the patient has been induced. A minimum $8.0 \mathrm{~mm}$ size ETT is recommended; and multiple attempts at inserting an ETT should be avoided. Neuromuscular agents are oftentimes used and should be reversed before extubation. For maintenance of anesthesia, a combination of IV and inhalational anesthetics is used. Short-acting opioids like remifentanil are preferred (4).

Establishing an airway is difficult in patients with severe CAO. Oftentimes they do not present with an upper airway obstruction where a tracheostomy or surgical airway can bypass the obstruction as the obstruction may be more distal. These patients need rigid bronchoscopy in order to deliver therapeutic interventions. An anesthetic strategy for rigid bronchoscopy is highlighted here by Conacher 
Table 1 ECMO indications for respiratory support

Acute respiratory distress syndrome
Severe bacterial or viral pneumonia
Aspiration syndromes
Alveolar proteinosis
Assistance to provide lung rest
Airway obstruction
Pulmonary contusion
Smoke inhalation
Lung transplant
Bridge to transplantation
Primary graft dysfunction
Intraoperative ECMO
Lung hyperinflation
Status asthmaticus
Pulmonary hemorrhage or massive hemoptysis
Congere air leak syndromes

ECMO, extracorporeal membrane oxygenation.

Table 2 Contraindications to ECMO use for respiratory support

Absolute: futile treatment without an exit strategy

Metastatic cancer

Known severe brain injury

Unwitnessed arrest

Prolonged CPR without adequate tissue perfusion

Severe chronic organ dysfunction

Cardiogenic failure or severe pulmonary hypertension precluding VV-ECMO use

Relative: contraindication to anticoagulation, advanced age, obesity

ECMO, extracorporeal membrane oxygenation; VV-ECMO, venovenous $\mathrm{ECMO}$; $\mathrm{CPR}$, cardiopulmonary resuscitation.

et al. In order to prepare the patient for rigid bronchoscope insertion into the trachea, using glycopyrrolate can offset potential vagal effects. Midazolam is administered followed by remifentanil infusion, and then a paralytic agent is given followed by propofol infusion. Once the patient is paralyzed, the rigid bronchoscope is inserted into the trachea to establish the airway and ventilation and oxygenation can be maintained through jet ventilation that is attached to the scope. Then, the IP is able to pass instruments freely through the scope in order to treat the $\mathrm{CAO}(5)$.

In severe cases of $\mathrm{CAO}$ when there is doubt that endotracheal intubation or rigid bronchoscopy can maintain adequate levels of ventilation and oxygenation, extracorporeal membrane oxygenation (ECMO) has been reportedly used in these scenarios. ECMO is conventionally used for refractory hypoxemic respiratory failure [i.e., severe acute respiratory distress syndrome (ARDS)] and for cardiac support. Large bore cannulas are placed in either venous or arterial vessels; blood is withdrawn from the venous circulation and into tubes connected to a centrifugal pump. The blood goes through an oxygenator where carbon dioxide is removed and the blood is reoxygenated. The reoxygenated blood is then returned to the patient. Patients without cardiac dysfunction can receive venovenous ECMO (VV-ECMO) whereas those with cardiac dysfunction receive venoarterial ECMO (VA-ECMO). The indications and contraindications to ECMO for respiratory support are outlined in Tables 1,2 (6).

ECMO can be used in the short term as a means of maintaining ventilation and oxygenation in severe CAO while therapeutic interventions are performed without worrying about securing an airway or airway fires in the setting of marked aberrations in oxygenation. Several case reports and case series have reported successful use of short term VV- or VA-ECMO as a bridge for airway support while severe CAOs are being therapeutically intervened upon. We highlight a few cases in the literature where $\mathrm{ECMO}$ has been used for $\mathrm{CAO}$ of various etiologies.

\section{Malignant and benign CAO}

In a case series of 18 patients described by Hong et al., the majority of patients had severe $\mathrm{CAO}$ due to malignancy; 2 had tracheal stenosis from prior intubations. These patients were supported by VV-ECMO and had interventions such as malignant mass removal, tracheal stent placement, tracheal stent removal, and mass debulking. Anticoagulation was given as an IV bolus of heparin (3,000-5,000 IU) during placement of the ECMO cannulas with 6 cases receiving continuous IV anticoagulation. Those that had cancer debulking only had the IV heparin bolus. The time spent on ECMO for these patients was a median time of 20.9 hours. All patients in the case series were successfully weaned off ECMO and decannulated. 
Four patients died of their underlying cancer. Reported complications were due to bleeding. One patient who had IV bolus heparin had massive hemorrhage after a tracheal stent was removed. Other bleeding complications include cannula site bleeding, gastrointestinal (GI) bleeding, intracranial bleeding and pulmonary hemorrhage. This case series showed that VV-ECMO may have a role in management of patients with severe $\mathrm{CAO}$ where life threatening airway compromise is a high possibility. VV-ECMO provides a bridge for ventilatory support and gives operators more time to be able to deliver therapeutic interventions on the stenosed area without concern of losing the airway. Bleeding complications are the most frequently encountered complication and using IV bolus heparin instead of continuous heparin infusion can minimize bleeding complications (7).

\section{Stent removal}

Another case report highlights the use of VV-ECMO during a tracheal stent removal. The stents were placed proximally in the trachea of a 27 -year-old man due to stenosis from a prior endotracheal intubation from a motor vehicle accident. Granulomatous tissue had obstructed part of the proximal portion of the stent and reduced the tracheal lumen to about $5 \mathrm{~mm}$ in diameter. Because of the difficulty with placing an ETT of that diameter and being able to successfully ventilate and oxygenate the patient, the decision was made to place him preemptively on VV-ECMO during the procedure. Once the cannulas were placed in the right internal jugular vein and right femoral vein, the patient was heparinized with IV bolus heparin and blood gases were checked through the ECMO circuit to ensure adequate oxygenation and ventilation. The IP then extracted the fragmented tracheal stents with bleeding during the stent extraction and lasering of granulomatous tissue and to obtain hemostasis. The patient was then transitioned to endotracheal intubation and then weaned from VV-ECMO and decannulated. The total time on VV-ECMO was about 120 minutes. He was extubated without complications shortly after the procedure (8).

\section{Stent placement}

A small retrospective analysis demonstrated the feasibility and safety of using VV ECMO during placement of selfexpandable metallic stents for benign and malignant obstructions. In this case series of 17 patients by Park et al. (9), 16 patients had critical airway obstruction due to malignancy and 1 was for a benign etiology. An ETT was able to be successfully inserted in 13 of the 17 patients. After a multidisciplinary decision between the cardiac surgeons, IPs, oncologists and radiologists, the decision was made to place these patients on VV-ECMO for stent placement in anticipation of ventilation difficulty by conventional means or if the patients could not lay flat. Anticoagulation was administered by IV heparin infusion for the majority of the patients and some had continuous anticoagulation. Patients were on VV-ECMO for a mean of 9 hours before stent procedure and had a mean total duration on ECMO for 42 hours. There was one cannula insertion complication with a right femoral vein rupture and subsequent hematoma formation and another had a superior femoral artery-femoral vein fistula formation that required a graft to repair it. Stent placement was successful in all patients and without procedural related complications. All patients were successfully weaned from ECMO and $85 \%$ of patients were extubated by post-operative day 6 of stent procedure. Two patients (12\%) were unable to be extubated and died of prolonged respiratory failure. Stent complications occurred at a rate of $30 \%$ with migration, fracture, collapse and sputum obstruction which were all managed by further interventional procedures.

The study by Park et al. demonstrated that VV-ECMO may be safely used as a means of maintaining adequate ventilation and oxygenation in a patient with critical airway obstruction. In these high-risk procedures, IPs feel more at ease knowing that the patient's airway is secured and can maintain ventilation while performing therapeutic procedures. All patients in the study were able to be weaned from ECMO and had a complication rate of $12 \%$ due to cannula complications. In rigid bronchoscopy, ventilation can normally be maintained with intermittent positive pressure ventilation via a side port or through high frequency jet or manual ventilation; in these high-risk patients, this study showed that ECMO could be a bridge to performing a lifesaving procedure. The patients in this study had a mean survival of 120 days post procedure which is likely longer than they would have survived had they not had any intervention. However, ECMO may not be considered cost-effective given the short life span at the time of intervention (9).

\section{Foreign body retrieval}

ECMO has also been reportedly used in management of foreign body removal in rare instances. Park et al. highlights 
the use of ECMO in foreign body removal in a small retrospective case series of three pediatric patients with a mean age of 18 months. The indications for ECMO were tenuous respiratory distress and potential loss of airway support due to tracheal obstruction of foreign object. In this case series, the pediatric patients either had near tracheal obstruction and were placed on ECMO to facilitate retrieval of the food particles or the patient had a relatively more distal food impaction that was obstructing the left main bronchi leading to refractory hypoxemia requiring ECMO initiation and clearance of food particles. There were no reported complications with bronchoscopy or ECMO. All patients in this case series were decannulated from ECMO the same day as their procedure or within 24 hours of cannulation. In all these patients, they were too unstable to undergo rigid bronchoscopy for retrieval of foreign body or the procedure would potentially create a near total occlusion of the airway. These patients presented with difficulty ventilating and oxygenating and ECMO was needed in order to rescue and facilitate retrieval. Without ECMO, these cases would have likely ended in death (10).

Lastly, case reports have highlighted the use of ECMO in setting of life-threatening hemoptysis. ECMO was used as a bridge for therapeutic intervention for a patient who had hemoptysis due to a left phrenic artery-pulmonary artery fistula in a patient with bronchiectasis. This systemicpulmonary artery shunt caused life-threatening hemoptysis. The patient developed refractory hypoxemia and was cannulated on ECMO as rescue therapy and was able to have a successful embolization procedure (11). Other case reports demonstrate using ECMO in setting of pulmonary hemorrhage due to pulmonary hypertension in a patient with biventricular heart failure requiring a left and right ventricular assist device (12).

In patients with life-threatening hemoptysis, the administration of an IV bolus or continuous infusion of heparin is not without risk. Recently, heparin-coated circuits and oxygen membranes have been developed. Heparin-free ECMO use has been described in case reports with both short term and more prolonged applications in thoracic trauma or traumatic brain injury. Ryu and colleagues describe its short-term use in a patient with significant chest trauma requiring surgical repair. A heparin free approach was maintained for 59 hours after initial cannulation (13). Both Muellenbach et al. and Biscotti et al. have described the prolonged use of ECMO in patients with ARDS and traumatic brain injury, outlining the experience with three and two cases respectively $(14,15)$. If a longer heparin-free approach is used, additional attention must be paid to the ECMO circuit to avoid excessive clot formation.

Complications of ECMO include equipment and tubing failure, cannula insertion difficulties, circuit thrombus, GI bleeding, sepsis and neurologic events. There have been case reports of patients who develop massive hemoptysis while on ECMO. In one systematic review evaluating anticoagulation strategies while on VV-ECMO, the reported rate of major hemorrhage (intracranial, GI bleeding, requirement of blood product or endoscopy, retroperitoneal bleeding, cannula site repair, or hemorrhagic shock) was approximately 16\%. Thrombotic events (clot requiring circuit change, deep vein thrombosis, pump head failure due to clot, or pulmonary embolism) happened at a rate of $53 \%$ (16). Pitcher et al. highlights how to manage hemoptysis while on ECMO (17). In a retrospective case series, 3 patients on VA-ECMO developed massive hemoptysis (defined as greater than $300 \mathrm{~mL}$ of blood in 24 hours) in the setting of antiplatelet therapy or a pulmonary artery catheter placement and were refractory to correction of coagulation, cold saline or epinephrine by bronchoscopy or bronchial blocker placement. The patients had their ETT clamped for up to 48 hours and if patients continued to bleed then they were referred to interventional radiology for bronchial artery embolization. It is imperative that while patients are off anticoagulation, the ECMO circuit and oxygenator are checked frequently for clot formation. Bronchoscopy is performed afterwards for removal of clot and bronchial casts. Cryotherapy can help facilitate removal of clot from the lungs (17). Once bleeding stops, anticoagulation is resumed due to thromboembolic risk with careful monitoring of rebleeding. Once the ETT is unclamped patients should be maintained on lung protective ventilation strategies (18).

It is important for physicians to be able to recognize the signs and symptoms of CAO. Oftentimes because of the slow growing nature of the stenotic airway lesion, by the time patients present to the hospital, they have already developed severe obstruction. Airway management is critical. Those presenting with respiratory distress and increased work of breathing but without evidence of hypoxemic or hypercapnic respiratory failure can initially be managed with heliox support while procedural planning takes place. Patients with CAO frequently undergo rigid bronchoscopy in order to therapeutically treat the obstruction with laser, balloon dilation, electrocautery, or stent placement. While these procedures are commonly performed by IPs with an anesthesiologist experienced with 
critical airway management, in rare instances when there is concern for the possibility of complete airway obstruction the procedure is escalated to include ECMO support. Experience with ECMO in CAO is limited to case reports and case series which highlight the successful short-term use of ECMO while performing therapeutic interventions. We summarized reports of ECMO used in patients with malignant and benign obstruction and foreign object removal. In all case reports, ECMO was preemptively initiated due to concern of an unstable airway. Physicians are more at ease when performing procedures while patients are receiving ECMO because even if there is full obstruction of the airway, ECMO is maintaining the patient's ventilation and oxygenation and they can maintain oxygenation during thermal ablation in patients otherwise requiring high fractions of inspired oxygen. Reported complications were rare and due to bleeding in setting of anticoagulation for ECMO or due to cannula complications. No complications were reported in the intraoperative period. While not often used, ECMO can be a safe management strategy in order to provide lifesaving interventions for a patient with critical CAO.

\section{Acknowledgments}

Funding: None.

\section{Footnote}

Provenance and Peer Review: This article was commissioned by the Guest Editors (Jonathan S. Kurman, Ashutosh Sachdeva and Rahul Nanchal) for the series "Interventional Pulmonology in the Intensive Care Unit Environment" published in Fournal of Thoracic Disease. The article has undergone external peer review.

Conflicts of Interest: Both authors have completed the ICMJE uniform disclosure form (available at: http://dx.doi. org/10.21037/jtd-2019-ipicu-08). The series "Interventional Pulmonology in the Intensive Care Unit Environment" was commissioned by the editorial office without any funding or sponsorship. Dr. Lin reports grants from the T32 HL007605 from the NIH/NHLBI during the conduct of the study. The other author has no conflicts of interest to declare.

Ethical Statement: The authors are accountable for all aspects of the work in ensuring that questions related to the accuracy or integrity of any part of the work are appropriately investigated and resolved.

Open Access Statement: This is an Open Access article distributed in accordance with the Creative Commons Attribution-NonCommercial-NoDerivs 4.0 International License (CC BY-NC-ND 4.0), which permits the noncommercial replication and distribution of the article with the strict proviso that no changes or edits are made and the original work is properly cited (including links to both the formal publication through the relevant DOI and the license). See: https://creativecommons.org/licenses/by-nc-nd/4.0/.

\section{References}

1. Ernst A, Feller-Kopman D, Becker HD, et al. Central airway obstruction. Am J Respir Crit Care Med 2004;169:1278-97.

2. Polaner DM. The use of heliox and the laryngeal mask airway in a child with an anterior mediastinal mass. Anesth Analg 1996;82:208-10.

3. Connolly KM, McGuirt WF Jr. Avoiding intubation in the injured subglottis: the role of heliox therapy. Ann Otol Rhinol Laryngol 2001;110:713-7.

4. Brodsky JB. Anesthetic considerations for bronchoscopic procedures in patients with central-airway obstruction. J Bronchol Interv Pulmonol 2001;8:36-43.

5. Conacher ID. Anaesthesia and tracheobronchial stenting for central airway obstruction in adults. Br J Anaesth 2003;90:367-74.

6. Makdisi G, Wang IW. Extra Corporeal Membrane Oxygenation (ECMO) review of a lifesaving technology. J Thorac Dis 2015;7:E166-76.

7. Hong Y, Jo KW, Lyu J, et al. Use of venovenous extracorporeal membrane oxygenation in central airway obstruction to facilitate interventions leading to definitive airway security. J Crit Care 2013;28:669-74.

8. Gourdin M, Dransart C, Delaunois L, et al. Use of venovenous extracorporeal membrane oxygenation under regional anesthesia for a high-risk rigid bronchoscopy. J Cardiothorac Vasc Anesth 2012;26:465-7.

9. Park JH, Shin JH, Kim KY, et al. Respiratory support with venovenous extracorporeal membrane oxygenation during stent placement for the palliation of critical airway obstruction: case series analysis. J Thorac Dis 2017;9:2599-607.

10. Park AH, Tunkel DE, Park E, et al. Management of complicated airway foreign body aspiration using extracorporeal membrane oxygenation (ECMO). Int J 
Pediatr Otorhinolaryngol 2014;78:2319-21.

11. Hsu SJ, Luo YH, Lee YC, et al. Life-threatening hemoptysis due to left inferior phrenic artery to pulmonary artery fistula rescued by extracorporeal membrane oxygenation therapy. Interact Cardiovasc Thorac Surg 2011;12:337-8.

12. Fukui S, Matsumiya G, Toda K, et al. Recovery from hemorrhagic pulmonary damage by combined use of a left ventricular assist system and right ventricular assist system and extracorporeal membrane oxygenation. J Heart Lung Transplant 2006;25:248-50.

13. Ryu KM, Chang SW. Heparin-free extracorporeal membrane oxygenation in a patient with severe pulmonary contusions and bronchial disruption. Clin Exp Emerg Med 2018;5:204-7.

14. Muellenbach RM, Kredel M, Kunze E, et al. Prolonged heparin-free extracorporeal membrane oxygenation in

Cite this article as: Lin J, Frye L. The intersection of bronchoscopy and extracorporeal membrane oxygenation. J Thorac Dis 2021;13(8):5176-5182. doi: 10.21037/jtd-2019-ipicu-08 multiple injured acute respiratory distress syndrome patients with traumatic brain injury. J Trauma Acute Care Surg 2012;72:1444-7.

15. Biscotti M, Gannon WD, Abrams D, et al. Extracorporeal membrane oxygenation use in patients with traumatic brain injury. Perfusion 2015;30:407-9.

16. Sklar MC, Sy E, Lequier L, et al. Anticoagulation Practices during Venovenous Extracorporeal Membrane Oxygenation for Respiratory Failure. A Systematic Review. Ann Am Thorac Soc 2016;13:2242-50.

17. Pitcher HT, Harrison MA, Shaw C, et al. Management considerations of massive hemoptysis while on extracorporeal membrane oxygenation. Perfusion 2016;31:653-8.

18. Nasim F, Nelson D, Schears G, et al. Bronchoscopic cryotherapy for bleeding on ECMO. Critical Care Medicine 2018;46:521. 